\title{
Laser-Induced Fluorescence of Rhenocene in Low-Temperature Matrices: Selective Excitation and Emission
}

\author{
Jeremy N. Hill, Robin N. Perutz,* and A. Denise Rooney ${ }^{1}$ \\ Department of Chemistry, University of York, York YOI SDD, U.K.
}

Received: June 20,1994

\begin{abstract}
Rhenocene, $\operatorname{Re}\left(\eta^{5}-\mathrm{C}_{5} \mathrm{H}_{5}\right)_{2}$, has been generated by photolysis of $\operatorname{Re}\left(\eta^{5}-\mathrm{C}_{5} \mathrm{H}_{5}\right)_{2} \mathrm{H}$ isolated in nitrogen and argon matrices at $12 \mathrm{~K}$, and has been studied by laser-induced fluorescence with a pulsed tunable laser. The emission lifetime of the lowest LMCT excited state is $(71.9 \pm 1.3) \mathrm{ns}$ in nitrogen and $(69.3 \pm 1.4) \mathrm{ns}$ in argon matrices. The emission and excitation spectra are far simpler and much better resolved than the UV/vis absorption spectrum, because the laser selects individual sites/conformers in the matrix (or groups of sites with very similar absorptions). Vibrational progressions are detected which are based on the ring-metal-ring stretching mode, $v_{4}$, and combinations of that mode with the symmetric ring breathing mode, $v_{2}$, and the symmetric $\mathrm{C}-\mathrm{H}$ deformation mode, $\nu_{3}$. In the LMCT excited state, there is an increase in $\nu_{4}$ of $5 \%$ and a decrease in $\nu_{3}(9 \%)$ and $\nu_{2}(3 \%)$, consistent with transfer of an electron from a ligand $\pi$-orbital to a metal-ligand bonding orbital.
\end{abstract}

\section{Introduction}

The sensitivity and selectivity of laser-induced fluorescence (LIF) makes it one of the most valuable of methods for obtaining structural information on small reactive molecules. ${ }^{2}$ We demonstrated several years ago that this technique could be applied to open-shell metallocenes isolated in low-temperature matrices. ${ }^{3-5}$ In the first experiments we used emission lines from a continuous wave (cw) laser to excite rhenocene, $\mathrm{ReCp}_{2}$ $\left(\mathrm{Cp}=\eta^{5}-\mathrm{C}_{5} \mathrm{H}_{5}\right)$, isolated in $\mathrm{CO}$ and $\mathrm{N}_{2}$ matrices. In this paper, we report the LIF of rhenocene excited with a pulsed, tunable dye laser. These experiments have enabled us to record both excitation and emission spectra which are selective for individual trapping sites or molecular conformers in the matrix. The resulting spectra are fully vibrationally resolved, allowing the determination of the totally symmetric vibrational frequencies in ground and excited states. Some of this work has been published as preliminary communications. ${ }^{6}$

Open-shell metallocenes with 16 and 17 valence electrons are not isolable for the second- and third-row transition metals but have been postulated as reaction intermediates in solution. ${ }^{5}$ However, the neutral metallocenes of Mo, W, and Re may be studied by photolysis of the corresponding hydride complexes $\mathrm{MCp}_{2} \mathrm{H}_{n}(\mathrm{M}=\mathrm{Mo}, \mathrm{W}, n=2 ; \mathrm{M}=\mathrm{Re}, n=1) .^{5}$ In previous work, we identified thenocene in nitrogen and carbon monoxide matrices by a combination of IR, UV/vis absorption, and emission spectroscopies and magnetic circular dichroism. ${ }^{3,7} \mathrm{We}$ showed that it has a parallel sandwich structure with an $\left(e_{2}\right)^{3}$ $\left(a_{1}\right)^{2}$ configuration, resulting in an orbitally degenerate, ${ }^{2} \mathrm{E}_{2}$, ground state, as is also found for ferricenium and many other 17-electron metallocenes. The visible absorption spectrum shows a prominent band with vibrational fine structure assigned to a LMCT transition ( $E_{5 / 2} \rightarrow E_{3 / 2}$ taking spin-orbit coupling into account). The major features of the progression are separated by ca. $340 \mathrm{~cm}^{-1}$. Within each vibrational component, there are a number of poorly resolved overlapping bands. We cannot distinguish the conformation of the rings from the spectra, and it is likely that more than one conformer is trapped in the matrix. Although metallocenes may adopt eclipsed $\left(D_{5 h}\right)$, staggered $\left(D_{5 d}\right)$, or intermediate $\left(D_{5}\right)$ structures, the point group has little impact on selection rules.

\footnotetext{
${ }^{\otimes}$ Abstract published in Advance ACS Abstracts, November 15, 1994.
}

Since the LMCT transition of rhenocene is fully allowed, the vibrational fine structure consists of progressions in totally symmetric vibrations. There are four totally symmetric fundamentals regardless of the conformation $\left(D_{5 d}, D_{5 h}\right.$, or $D_{5}$ point groups): ${ }^{8-10} v_{1}, \mathrm{C}-\mathrm{H}$ stretch (ca. $3000 \mathrm{~cm}^{-1}$ ); $v_{2}$, ring breathing mode $\left(1101 \mathrm{~cm}^{-1}\right) ; \nu_{3}$, perpendicular $\mathrm{C}$ - $\mathrm{H}$ deformation $(830$ $\left.\mathrm{cm}^{-1}\right) ; v_{4}$, ring-M-ring stretching mode $\left(326 \mathrm{~cm}^{-1}\right)$. These ground-state frequencies are taken from the earlier LIF experiments with $\mathrm{cw}$ excitation (other than for $v_{1}$ ). Here, we use the same numbering as Aleksanyan. ${ }^{10}$ Even overtones of nontotally-symmetric vibrations should also satisfy the conditions for the vibrational progression. ${ }^{11}$ In practice, the vibrational structure arises principally from the ring-metal-ring symmetric stretching mode, $v_{4}$, though $v_{2}$ and $v_{3}$ also contribute.

Apart from our own work, there have been two groups of studies of emission from cyclopentadienyl complexes, both reporting extensive vibrational fine structure. The emission of ruthenocene in the solid state has been assigned to a spinforbidden ligand-field transition: ${ }^{12-15}$ much improved spectra have been reported recently with laser excitation. ${ }^{15,16}$ Miller et al. have employed LIF to study monocyclopentadienyl complexes, $\mathrm{MCp}(\mathrm{M}=\mathrm{Zn}, \mathrm{Cd}, \mathrm{Mg}, \mathrm{Ca})$, in the gas phase. ${ }^{17,18}$ The importance of emission spectroscopy for organometallics is increasingly recognized, ${ }^{19 a}$ and more examples of emission from LMCT states are appearing. ${ }^{19 \mathrm{~b}}$ However, the use of laser excitation remains very limited.

\section{Experimental Section}

1. $\operatorname{ReCp}_{2} \mathrm{H}$. The precursor, $\mathrm{ReCp}_{2} \mathrm{H}$, was handled by using Schlenk and glovebox techniques. It was synthesized from $\mathrm{ReCl}_{5}, \mathrm{NaCp}$, and $\mathrm{NaBH}_{4}{ }^{20}$

2. Matrix Equipment. Matrix isolation experiments were conducted at the Rutherford-Appleton Laboratory with a mobile rig incorporating three trolleys, one each for the closed-cycle cooler, the main pumps, and the glass vacuum line. Fine movements of the cold tip are achieved by using a three-way translation mount. The Displex expander (Air Products CS202) is inserted via a double $O$-ring seal into a stainless steel vacuum shroud attached to the translation mount. Quartz windows are fixed to three of the four vertical surfaces of the shroud and to the base via O-ring seals. The central window, on which the matrix is deposited, is made from $\mathrm{BaF}_{2}$ and is held in a canted 
copper mount (ca. $40^{\circ}$ to vertical). $\mathrm{ReCp}_{2} \mathrm{H}$ was evaporated from a right-angle tube and codeposited with the matrix gas onto the window at $20 \mathrm{~K}$ (temperature monitored with a hydrogen vapor bulb). The glass tube and the inlet for matrix gas occupy the fourth vertical surface of the shroud. The $\mathrm{ReCp}_{2} \mathrm{H}$ was deposited for $88-125$ min with the tube held at 307 (dilute matrices) $-316 \mathrm{~K}$ (concentrated). After deposition the matrices were cooled slowly to $12 \mathrm{~K}$ and photolyzed for 47-135 min with an Applied Photophysics 200-W mercury arc fitted with a $5-\mathrm{cm}$ water filter. Matrices were used for up to a week without degradation in spectroscopic quality.

The main pumping trolley evacuates the matrix shroud to $1 \times 10^{-6}$ mbar prior to cool-down and $1 \times 10^{-7}$ mbar after cooling. The glass vacuum line was evacuated to $5 \times 10^{-4}$ mbar. Dinitrogen and argon gases used for matrices were BOC research grade (99.999\%). Gases were passed through a liquid nitrogen-cooled spiral trap (to remove water vapor) placed after the needle valve at a rate of about $2 \mathrm{mmol} \mathrm{h}^{-1}$.

3. Laser, Optics, and Sampling. A Lumonics Hyperex 460 $\mathrm{XeCl}$ excimer laser $(308 \mathrm{~nm}, 150 \mathrm{~mJ}$ per pulse) was used to pump a Lambda-Physik FL3002 dye laser (output: pulse width $10 \mathrm{~ns}$, linewidth $<0.1 \mathrm{~cm}^{-1}$ ). The output beam passed through two quartz steering prisms to a variable neutral density filter, which allowed continuous variation of the laser pulse energy. The latter consisted of two prismatic troughs (isosceles triangle, unique angle ca. $6^{\circ}$; Hellma Ltd.), which could be moved synchronously in opposite directions and were filled with aqueous $\mathrm{NiSO}_{4}$ and $\mathrm{CoSO}_{4}$. A quartz flat acted as a beam splitter, reflecting ca. $10 \%$ of the beam energy onto the vacuum photodiode (ITL Instrument Technology with an EMI PM28B power supply), which was used to monitor the laser pulse energy. The beam was raised with a periscope arrangement of prisms in conjunction with two irises and and entered the sample area vertically upward where it was focused by a $f=80 \mathrm{~mm}$ lens onto the matrix. Care was taken not to focus the beam too tightly to avoid damage to the matrix. Emission was collected at $90^{\circ}$ to the exciting source, with a $f=150 \mathrm{~mm}$ collecting lens focusing onto the entrance slit of the Spex 1877 Triplemate, which was set to give $5-\mathrm{cm}^{-1}$ resolution and ca. 20-nm spectral window.

4. Detector, Gating, and Timing System. The emission was detected on a IRY700 OSMA intensified diode array (Spectroscopic Instruments $\mathrm{GmbH}$ ), cooled to $-30^{\circ} \mathrm{C}$. Atomic lines were used for wavelength calibration and to measure the detector resolution. The OSMA was used in continuous wave (cw) mode for recording lamp spectra and in gated mode for LIF. Data collection and manipulation was handled by using dedicated "POSMA" software on a PC. The gate on the detector was produced by a Princeton Instruments FG100 pulse generator. The gate length was typically set at $100 \mathrm{~ns}$ and initially was positioned centrally with respect to the laser pulse, as viewed on the oscilloscope (Tektronix 2467, $350 \mathrm{MHz}$ ). A second pulse/delay generator (Stanford Research Systems DG535) controlled the laser firing and repetition rate (normally $10 \mathrm{~Hz}$ ) and set the delays between the laser pulse and the gate. At the start of the experiment, the gate and laser pulse were synchronized. They were then monitored throughout the experiment.

5. Measurement of Laser Energy. Absolute laser energies (Gentech power meter) were less than $100 \mu \mathrm{J}$ per pulse. A relative measure of the laser energy was obtained for each LIF spectrum with the vacuum photodiode and a Stanford Research Systems SRS 250 gated integrator (boxcar) coupled to a SRS 280 display. The boxcar was triggered by the DG535 pulse generator. When the boxcar gate and laser pulse overlapped, it displayed a signal for each laser shot and a moving average of a selected number $(10,20,100$, or 1000$)$ of the preceding shots. If the energy drifted significantly during a run, the run was repeated. The laser output fluctuated typically by $\leq 5 \%$ of the mean value.

6. General Procedures for Recording Spectra. Initially, the fluorescence signal was maximized in real time (update ca. $1 \mathrm{~Hz}$ ), adjusting the detector gate with respect to the laser pulse. The number of exposures for an actual spectrum was set to use the full range of the detector (maximum number of counts per scan ca. 16 000). Each exposure lasted $33 \mathrm{~ms}$. A background spectrum, recorded under identical timing conditions, but with the beam to the sample blocked and the slits open, was subtracted from each set of scans. The signal to noise ratio was improved by averaging a number of sets of exposures (typically 50-2000 exposures averaged up to 20 times, total exposure time 1.7-132 s, total collection time 5-800 s). Energy dependence plots were recorded for each sample by varying the path length of the laser through the neutral density prism. The linearity of emission intensity with increasing laser energy confirmed that only single-photon processes were observed under the conditions employed.

For emission spectra, each scan covered ca. 20-nm spectra width, so that complete fluorescence spectra were recorded in three or four segments. The spectra were wavelength-calibrated with at least two atomic emission lines of neon, krypton, or mercury (error ca. $\pm 2 \mathrm{~cm}^{-1}, 0.05 \mathrm{~nm}$ ).

Excitation spectra were recorded with a single detection window. At least three readings of the boxcar were made to obtain a precise measure of the laser energy. A complete set of spectra for an excitation profile required several days to record and often involved using more than one laser dye. To ensure that the intensity distribution in excitation was as precise as possible, the emission spectra around each excitation peak were recorded in one session. Emission spectra obtained from different sessions were scaled appropriately by comparison of sample spectra collected at common excitation wavelengths. The excitation spectra were calculated by dividing the counts at a chosen emission wavelength by the boxcar reading (see Figures 4 and 8 ).

Decay kinetics were recorded by increasing the delay on the gate signal relative to the laser pulse with a single observation window. With the gate length set at $100 \mathrm{~ns}$, the delay was increased in steps of $10 \mathrm{~ns}$.

\section{Results}

1. Rhenocene in Nitrogen Matrices. Rhenocene was generated by broad-band UV photolysis of matrix-isolated $\mathrm{ReCp}_{2} \mathrm{H}$ and probed by low-energy pulses from an excimerpumped dye laser. The emission was invariably detected after monochromation on a multichannel detector. Rhenocene absorbs between 440 and $490 \mathrm{~nm}$ and emits from 485 to 560 $\mathrm{nm} .^{3}$ The LIF spectrum generated by pulsed laser irradiation at $473.5 \mathrm{~nm}$ is displayed as Figure 1a (with the spectrum in an argon matrix shown as Figure 1b). This spectrum is practically identical to that recorded with $\mathrm{cw}$ laser excitation. ${ }^{3}$ Unlike the absorption spectrum, single sharp bands are detected. The electronic origin of the transition, $T_{0}$, is precisely located from the emission spectrum. The bandwidth of each vibrational feature is such that $\nu_{2}{ }^{\prime \prime}$ and $\nu_{3}{ }^{\prime \prime}$ can be measured, and most of the vibrational components may be fitted to combinations of the three lowest totally symmetric modes: $v_{4}^{\prime \prime}, v_{2}^{\prime \prime}, v_{3}^{\prime \prime}$ (Table 1). The dominant progression carrying mode is $v_{4}^{\prime \prime}$ with overtones detected up to $v=8$, though there may be some ambiguity in the assignment of the higher overtones. At longer 


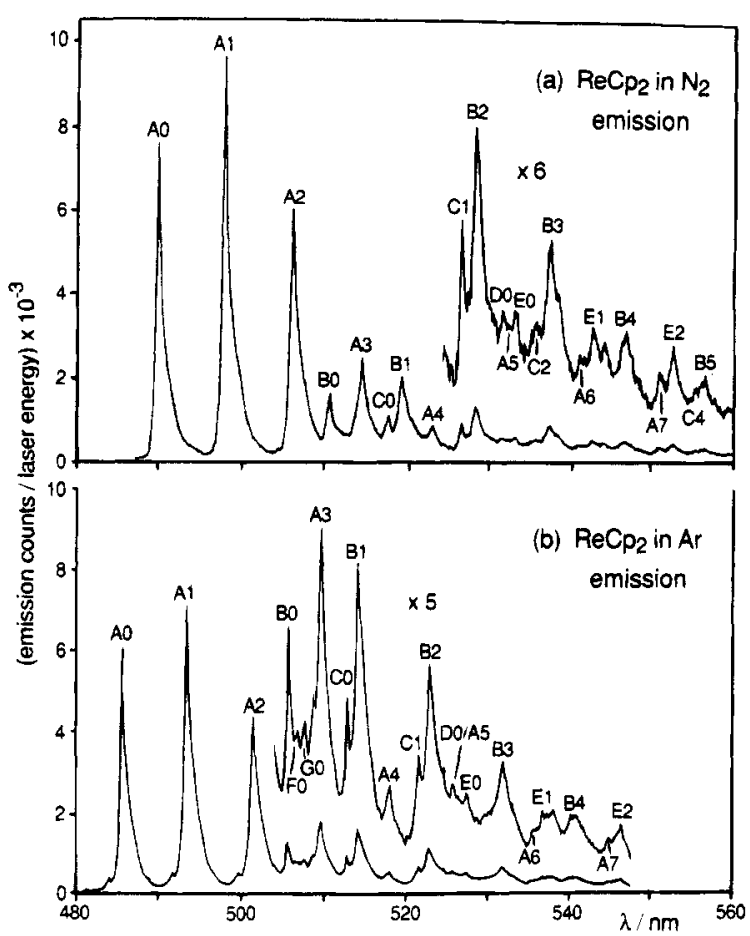

Figure 1. Emission spectrum of $\mathrm{ReCp}_{2}$ obtained following UV photolysis of $\mathrm{ReCp}_{2} \mathrm{H}$ (a) in a nitrogen matrix at $12 \mathrm{~K}$ excited with $\lambda_{\text {ex }}$ $=473.5 \mathrm{~nm}$ (each progression is indicated by a different letter, Table 1) and (b) in an argon matrix at $12 \mathrm{~K}, \lambda_{\mathrm{ex}}=470.0 \mathrm{~nm}$ (band positions and assignments in Table $\mathrm{V}$, supplementary material).

TABLE 1: Emission for $\operatorname{ReCp}_{2}$ in a Nitrogen Matrix, $\lambda_{\mathrm{ex}}=$ 473.5 nm, "Sharp" Site (See Figure 1a)

\begin{tabular}{|c|c|c|c|c|c|}
\hline$\lambda, \mathrm{nm}$ & $\bar{v}, \mathrm{~cm}^{-1}$ & $\begin{array}{c}\Delta \bar{\nu}, \mathrm{cm}^{-1} \\
\text { (from origin) }\end{array}$ & label & assgt & $\begin{array}{l}\text { interval, } \\
\mathrm{cm}^{-1}\end{array}$ \\
\hline 489.79 & 20417 & 0 & A0 & $T_{0}$ & 0 \\
\hline 497.74 & 20091 & 326 & Al & $v_{4}^{\prime \prime}$ & $T_{0}-326$ \\
\hline 505.94 & 19765 & 652 & A2 & $2 \nu_{4}^{\prime \prime}$ & $A 1-326$ \\
\hline 510.49 & 19589 & 828 & B0 & $v_{3}^{\prime \prime}$ & $T_{0}-828$ \\
\hline 514.37 & 19441 & 976 & A3 & $3 v_{4}^{\prime \prime}$ & $A 2-324$ \\
\hline 517.67 & 19317 & 1100 & $\mathrm{CO}$ & $v_{2}^{\prime \prime}$ & $T_{0}-1100$ \\
\hline 519.22 & 19260 & 1157 & B1 & $v_{3}^{\prime \prime}+v_{4}^{\prime \prime}$ & B $0-329$ \\
\hline 523.01 & 19120 & 1297 & A4 & $4 v_{4}^{\prime \prime}$ & $A 3-321$ \\
\hline 526.49 & 18994 & 1423 & $\mathrm{Cl}$ & $v_{2}^{\prime \prime}+v_{4}^{\prime \prime}$ & $\mathrm{CO}-324$ \\
\hline 528.17 & 18936 & 1481 & B2 & $v_{2}^{\prime \prime}+2 v_{4}^{\prime \prime}$ & B1 -324 \\
\hline 531.4 & 18818 & 1599 & D0 & & $\mathrm{T}_{0}-1599$ \\
\hline 532.1 & 18793 & 1624 & A5 & $5 \nu_{4}^{\prime \prime}$ & $A 4-327$ \\
\hline 532.9 & 18765 & 1652 & EO & $2 v_{3}^{\prime \prime}$ & $T_{0}-1652$ \\
\hline 534.9 & 18695 & 1722 & & & \\
\hline 535.6 & 18671 & 1746 & $\mathrm{C} 2$ & $v_{2}^{\prime \prime}+2 v_{4}^{\prime \prime}$ & $\mathrm{C} 1-323$ \\
\hline 537.3 & 18612 & 1805 & B3 & $v_{3}^{\prime \prime}+3 v_{4}^{\prime \prime}$ & B2 -324 \\
\hline 538.1 & 18584 & 1833 & & & \\
\hline 541.4 & 18471 & 1946 & A6 & $6 v_{4}^{\prime \prime}$ & A5 - 322 \\
\hline 542.5 & 18433 & 1984 & E1 & $2 v_{3}^{\prime \prime}+v_{4}^{\prime \prime}$ & $E 0-332$ \\
\hline 543.9 & 18386 & 2031 & & & \\
\hline 546.6 & 18295 & 2122 & B4 & $v_{3}^{\prime \prime}+4 v_{4}^{\prime \prime}$ & B3 -317 \\
\hline 550.6 & 18162 & 2255 & A7 & $7 v_{4}^{\prime \prime}$ & A6 -309 \\
\hline 552.4 & 18103 & 2314 & E2 & $2 v_{3}^{\prime \prime}+2 v_{4}^{\prime \prime}$ & E1 -330 \\
\hline 555.2 & 18012 & 2405 & $\mathrm{C} 4$ & $v_{2}^{\prime \prime}+4 v_{4}^{\prime \prime}$ & \\
\hline 555.9 & 17989 & 2428 & & & \\
\hline 556.3 & 17976 & 2441 & B5 & $v_{3}^{\prime \prime}+5 v_{4}^{\prime \prime}$ & B4 - 319 \\
\hline 560.3 & 17848 & 2569 & A8 & $8 v_{4}^{\prime \prime}$ & A7 -315 \\
\hline 562.1 & 17790 & 2627 & E3 & $2 v_{3}{ }^{\prime \prime}+3 v_{4}^{\prime \prime}$ & $\mathrm{E} 2-313$ \\
\hline 563.0 & 17762 & 2655 & & & \\
\hline
\end{tabular}

wavelengths the spectra become complicated by combinations of $\nu_{2}^{\prime \prime}$ and $v_{3}^{\prime \prime}$ with $\nu_{4}^{\prime \prime}$.

The intensity of fluorescence varies linearly with the laser energy (Figure 2), demonstrating that the emission arises from a single-photon process. The effect on the emission spectrum of $\mathrm{ReCp} \mathrm{p}_{2}$ upon changing the laser wavelength is shown in Figure 3. The chosen excitation wavelengths excite into the second

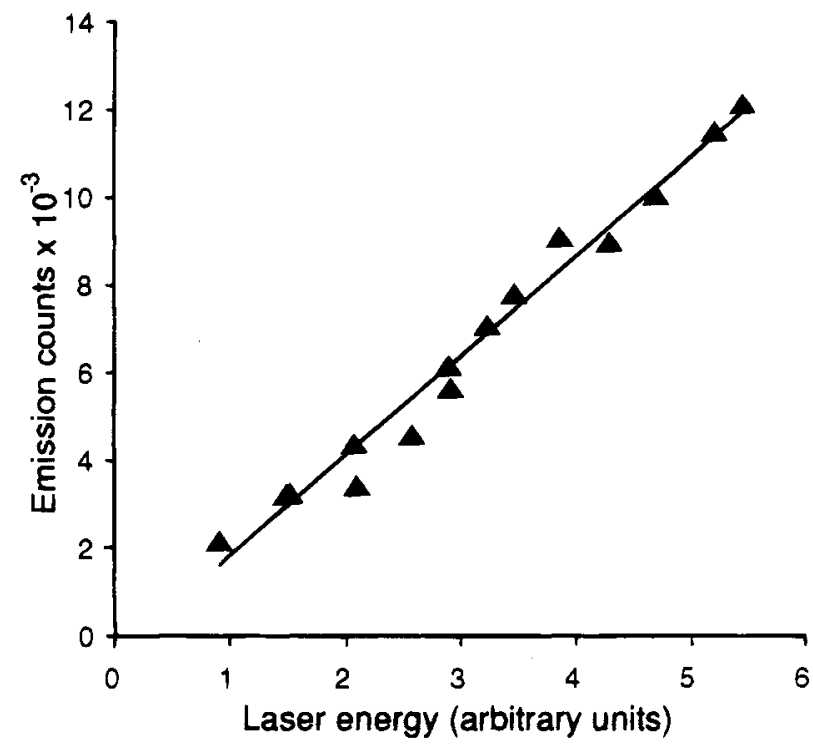

Figure 2. Plot showing the linearity of fluorescence intensity with increasing laser energy for $\mathrm{ReCp}_{2}$ in a nitrogen matrix, $\lambda_{\text {ex }}=473.5$ $\mathrm{nm}$. The laser energy corresponds to the reading on the boxcar.

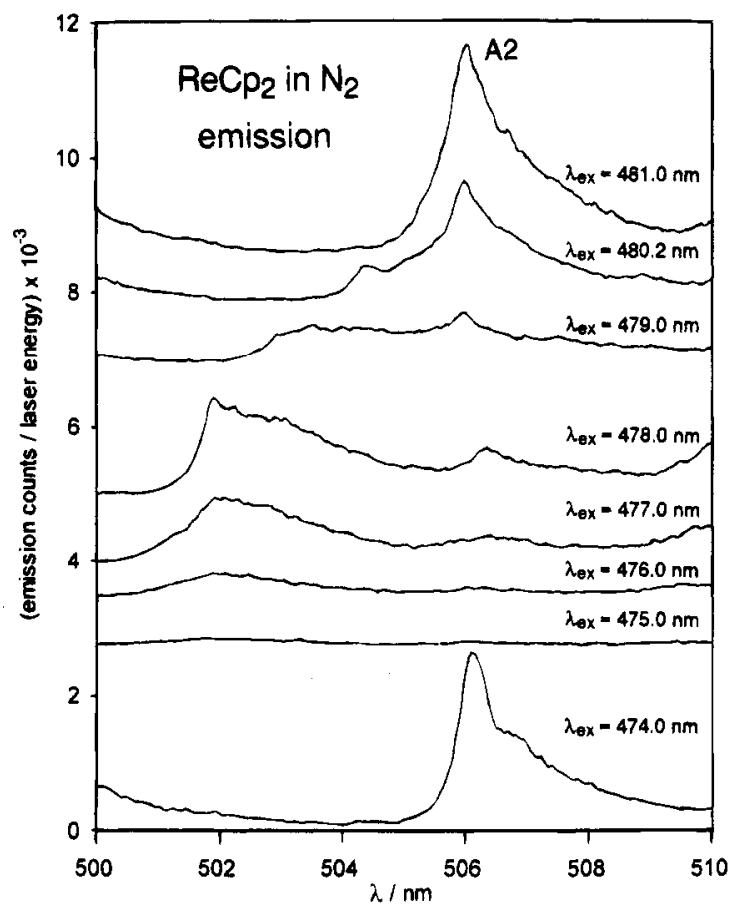

Figure 3. Expanded emission spectra of the $(0,2)$ transition of $\mathrm{ReCp}_{2}$ in a nitrogen matrix, showing the effect of excitation wavelength on the resultant emission.

and third vibrational components of the absorption spectrum (see Figure 4a). With $\lambda_{\mathrm{ex}}=481.0 \mathrm{~nm}$, sharp intense emission is seen at $\lambda_{\mathrm{em}}=506.0 \mathrm{~nm}$ (Figure 3 , top spectrum, $\lambda_{\mathrm{em}}$ denotes an emission wavelength; $\lambda_{\text {ex }}$ denotes an excitation wavelength). This feature (A2) is also observed in Figure 1a. Shortening the excitation wavelength reduces the intensity of this peak, while a weaker and much broader emission grows in at $\lambda_{\mathrm{em}}=$ $504.4 \mathrm{~nm}\left(\lambda_{\mathrm{ex}}=480.2 \mathrm{~nm}\right)$. The broad emission moves to shorter wavelengths as the laser wavelength is further reduced. The maximum intensity of this band is reached with $\lambda_{\mathrm{ex}}=478.0$ $\mathrm{nm}\left(\lambda_{\mathrm{em}}=502.1 \mathrm{~nm}\right)$. At $\lambda_{\mathrm{ex}}=475.0 \mathrm{~nm}$, practically no structured emission is seen: the position of the laser wavelength corresponds to the "trough" between the second and third vibrational components in the absorption spectrum (Figure 4a). However, further reduction of the excitation wavelength by just $1 \mathrm{~nm}$ induces emission very similar to that observed when $\lambda_{\mathrm{ex}}$ 


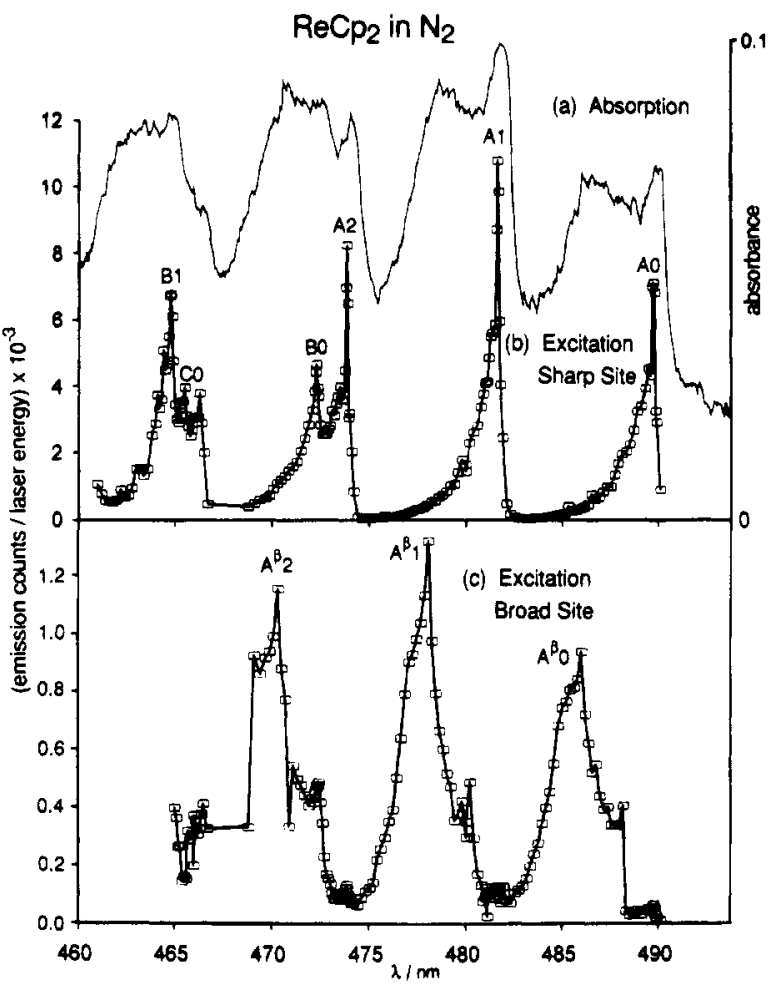

Figure 4. Spectra of $\mathrm{ReCp}_{2}$ in a nitrogen matrix. (a) absorption spectrum; (b) excitation spectrum for the "sharp" site measured from the intensity of the Al emission band $\left(\lambda_{\mathrm{em}}=497.7 \mathrm{~nm}\right)$; (c) excitation spectrum for the "broad" site measured from the intensity of the $\mathrm{A} 2^{\beta}$ "broad" emission band, $\lambda_{\text {em }}=502.3 \mathrm{~nm}$ (see Table 2).

$=481.0 \mathrm{~nm}$ (Figure 3, bottom spectrum). At $\lambda_{\mathrm{ex}}=474.0 \mathrm{~nm}$, the third member of the $\nu_{4}^{\prime}$ progression in the absorption spectrum is being probed at a position within the absorption band envelope equivalent to excitation of the second member with $\lambda_{\mathrm{ex}}=481.0 \mathrm{~nm}$. Hence the emission spectrum observed is virtually the same as with $\lambda_{\mathrm{ex}}=481.0 \mathrm{~nm}$, though it has a different intensity. These changes in the emission spectrum must reflect changes in the sites/conformers which are being probed by the laser. Therefore, the fine structure seen in the absorption spectrum originates in a number of matrix sites or conformers of rhenocene, one of which can be excited particularly selectively by the laser.

Since a laser has a very narrow bandwidth and can cause emission predominantly from a single site, an excitation spectrum of the "sharp" site seen in the fluorescence spectra of $\mathrm{ReCp}_{2}$ should be more highly resolved than the absorption spectrum. This indeed proves to be the case. Figure $4 \mathrm{a}$ is the expanded part of the absorption spectrum of $\operatorname{ReCp}_{2}$, which corresponds to the excitation spectrum below (Figure $4 \mathrm{~b}$ ). This excitation spectrum is recorded point-by-point, measuring the emission intensity of the A1 peak ( $\left.\lambda_{\mathrm{em}}=497.7 \mathrm{~nm}\right)$ as a function of laser wavelength and energy; unlike many excitation spectra, it does not monitor total emission. The excitation shows a sharp onset on the long-wavelength side with a longer tail to the blue of each vibrational feature. The $(0,0)$ bands of excitation and emission differ by only $2 \mathrm{~cm}^{-1}$; i.e., they are coincident within experimental error. There is a dramatic improvement in the resolution of the excitation spectrum compared to the absorption spectrum, enabling us to measure the frequencies of three symmetric modes in the excited state (Table 2).

We now consider the "broad" component of the emission spectrum. Excitation at $476.5 \mathrm{~nm}$ leads predominantly to broad emission, with a minor contribution from the sharp part. Although we did not record a full emission spectrum with pulsed excitation, a LIF spectrum with $\mathrm{cw}$ irradiation has been
TABLE 2: Excitation Maxima of the A1 Emission Band of $\mathrm{ReCp}_{2}$ in a Nitrogen Matrix, "Sharp" Site and the $A^{\beta 2}$ "Broad" Emission Band

\begin{tabular}{|c|c|c|c|c|c|}
\hline$\lambda, \mathrm{nm}$ & $\bar{v}, \mathrm{~cm}^{-1}$ & $\begin{array}{c}\Delta \bar{\nu}, \mathrm{cm}^{-1} \\
\text { (from origin) }\end{array}$ & label & assgt & $\begin{array}{l}\text { interval, } \\
\mathrm{cm}^{-1}\end{array}$ \\
\hline \multicolumn{6}{|c|}{ Sharp Site } \\
\hline 489.75 & 20419 & 0 & AO & $T_{0}$ & \\
\hline 481.65 & 20762 & 343 & $\mathrm{~A} 1$ & $v_{4}^{\prime}$ & $\mathrm{A} 0+343$ \\
\hline 473.85 & 21104 & 685 & A2 & $2 v_{4}^{\prime}$ & $A 1+342$ \\
\hline 472.30 & 21173 & 754 & B0 & $v_{3}^{\prime}$ & $\mathrm{T}_{0}+754$ \\
\hline 466.30 & 21445 & 1026 & A3 & $3 v_{4}^{\prime}$ & $\mathrm{A} 2+341$ \\
\hline 465.50 & 21482 & 1063 & $\mathrm{CO}$ & $v_{2}^{\prime}$ & $\mathrm{T}_{0}+1063$ \\
\hline 464.80 & 21515 & 1096 & B1 & $\nu_{3}^{\prime}+\nu_{4}^{\prime}$ & $\mathrm{B} 0+342$ \\
\hline \multicolumn{6}{|c|}{ Broad Site } \\
\hline 485.9 & 20580 & 0 & $\mathrm{~A}^{\beta} 0$ & $T_{0}$ & \\
\hline 478.0 & 20921 & 341 & $\mathrm{~A}^{\beta} 1$ & $v_{4}^{\prime}$ & $\mathrm{A}^{\beta} 0+341$ \\
\hline 470.3 & 21263 & 683 & $\mathrm{~A}^{\beta_{2}}$ & $2 v_{4}^{\prime}$ & $A^{\beta} 1+342$ \\
\hline
\end{tabular}
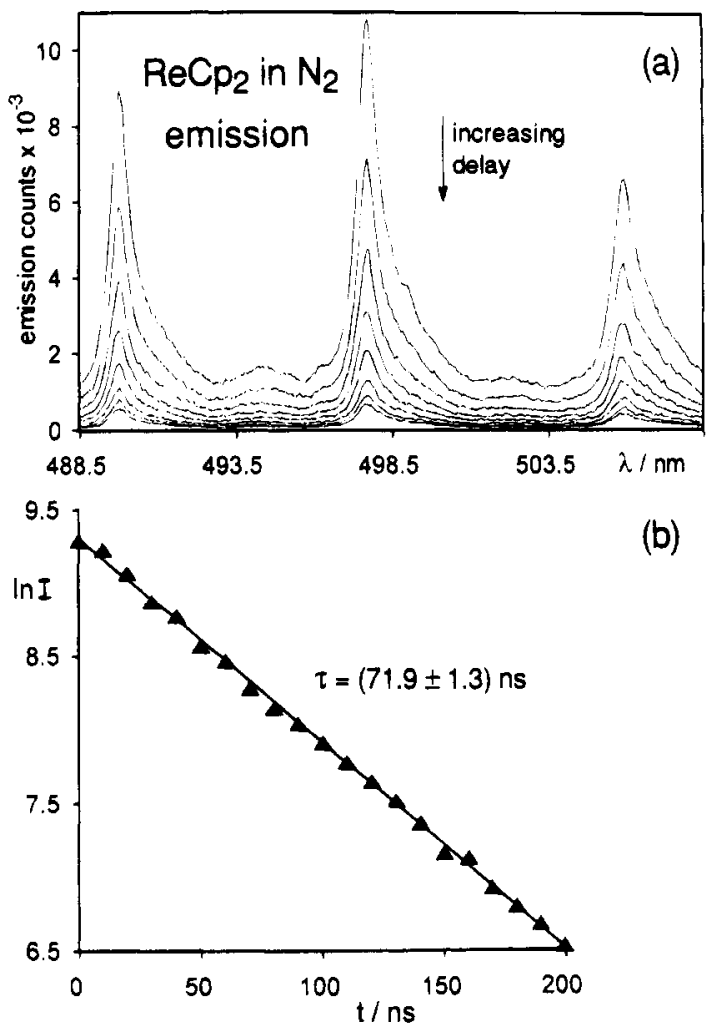

(b)

Figure 5. Emission spectra of $\mathrm{ReCp}_{2}$ in a nitrogen matrix. The delay between excitation pulse and observation is increased by $30 \mathrm{~ns}$ between each trace $\left(\lambda_{\text {ex }}=457.9 \mathrm{~nm}\right)$. (b) Logarithmic plot of the intensity of the emission of the A1 peak $\left(\lambda_{\mathrm{em}}=497.7 \mathrm{~nm}\right)$, yielding the fluorescence lifetime, $\tau$.

measured (Table III, supplementary material). The progressions are labeled with a superscript $\beta$ (e.g., $\mathrm{A}^{\beta} 0$ ) to distinguish them from the sharp progression. The $(0,0)$ band of the broad component is shifted by $154 \mathrm{~cm}^{-1}$ to higher energy compared to the sharp one. Progressions in $\nu_{4}{ }^{\prime \prime}$ are conspicuous, as is the combination with $\nu_{3}^{\prime \prime}$. However, $\nu_{2}^{\prime \prime}$ is not apparent. An excitation spectrum of the $A^{\beta} 2$ emission band is displayed as Figure $4 c$ with the assignments shown in Table 2.

Measurement of the emission intensity at a series of time delays after the laser pulse allows the decay kinetics of the excited state to be measured (Figure 5). Figure 5a shows part of the "sharp" emission spectrum, recording how the intensity falls off when observed at progressively longer delays after the excitation pulse. These spectra demonstrate that the rate of decay is the same at different emission wavelengths. Analysis of the decay reveals it to be first order, giving an excited state lifetime $\tau=71.9 \pm 1.3 \mathrm{~ns}$ (Figure $5 \mathrm{~b}$ ). A determination of the 

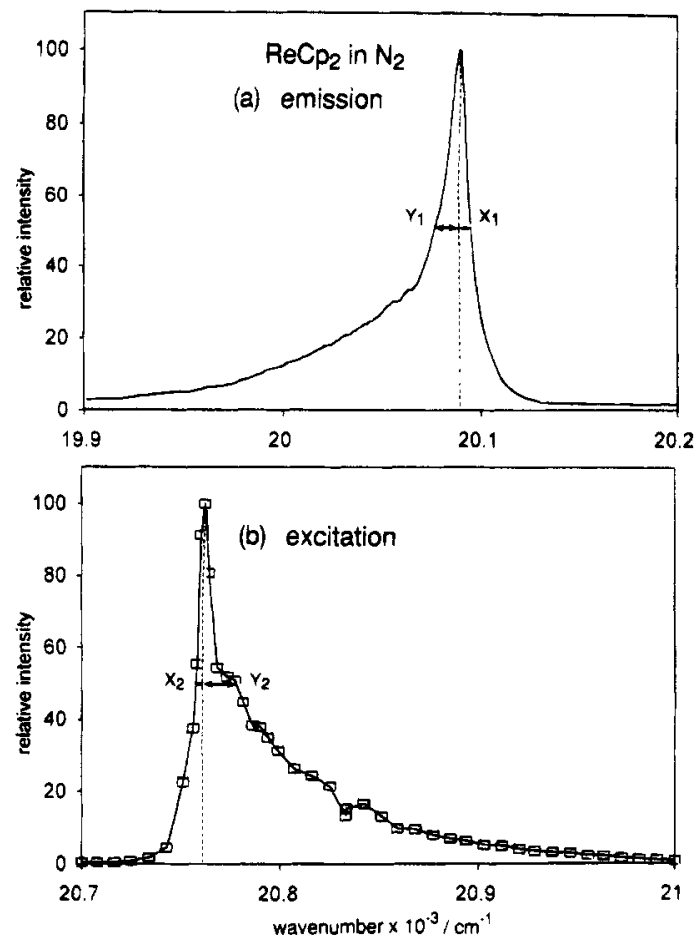

Figure 6. Comparison of the band shape for emission and excitation spectra of $\mathrm{ReCp}_{2}$ in a nitrogen matrix in the sharp site. Note that the spectra are plotted on a scale linear in energy. (a) The A2 emission band with $\lambda_{\mathrm{ex}}=473.85 \mathrm{~nm}$. $X_{1}$ and $Y_{1}$ are the half-widths at halfmaximum intensity of the band; $X_{1}=6 \mathrm{~cm}^{-1}, Y_{1}=13 \mathrm{~cm}^{-1}$. (b) The A2 excitation band: $X_{2}=5 \mathrm{~cm}^{-1} ; Y_{2}=15 \mathrm{~cm}^{-1}$.

lifetime of the "sharp" site in a more dilute matrix yielded $\tau=$ $71.0 \pm 2.7 \mathrm{~ns}\left(\lambda_{\mathrm{ex}}=473.5 \mathrm{~nm}\right.$ ), indicating that this lifetime is independent of metallocene concentration. The lifetime of the broad component in a dilute matrix is marginally longer ( $83 \pm$ $6 \mathrm{~ns}$ ) than that of the sharp one. At twice the standard error, the difference in the values of $\tau$ is barely significant.

The unusual line shapes of emission and excitation bands are illustrated as parts a and $b$ of Figure 6 for the $(0,1)$ and $(1,0)$ bands, respectively. The emission band exhibits a sharp onset with a long tail at lower energy. The excitation band is essentially the mirror image with the tail on the high-wavenumber side. The line shape remains the same for higher overtones in the excitation and emission spectra.

2. Rhenocene in Argon Matrices. It was concluded from the original IR experiments that $\mathrm{ReCp}_{2} \mathrm{H}$ was photochemically active in $\mathrm{CO}$ and nitrogen matrices but inert in argon matrices. ${ }^{3}$ In a more recent experiment with $\mathrm{ReCp}_{2}$ in $\mathrm{Ar}$, we have detected several features in the IR consistent with $\mathrm{ReCp}_{2}$, though they are extremely weak $\left(1101 \mathrm{~cm}^{-1}, \nu_{9} ; 995 \mathrm{~cm}^{-1}, \nu_{19}\right)$. However, there is no difficulty in detecting $\mathrm{ReCp}_{2}$ in argon by visible absorption (Table IV, supplementary material). The $(0,0)$ band in argon is shifted to higher energy by ca. $300 \mathrm{~cm}^{-1}$ compared to that in nitrogen. The vibrational spacing is consistent with a progression in $v_{4}{ }^{\prime}$. Each vibrational feature is broadened by fine structure, as is observed in nitrogen matrices, but it appears to be more complicated in argon matrices.

Laser irradiation at $470.0 \mathrm{~nm}$ leads to intense structured emission (Figure 1b; Table V, supplementary material). The appearance of the emission spectrum is similar to that observed in nitrogen matrices. The main progression is assigned out to the seventh overtone, although, as in nitrogen matrices, assignment at higher overtones is less certain. Progressions containing $\nu_{2}{ }^{\prime \prime}$ and $\nu_{3}^{\prime \prime}$ are observable. The peaks labeled F0 and G0 do not correspond to any symmetric modes and have no counterpart

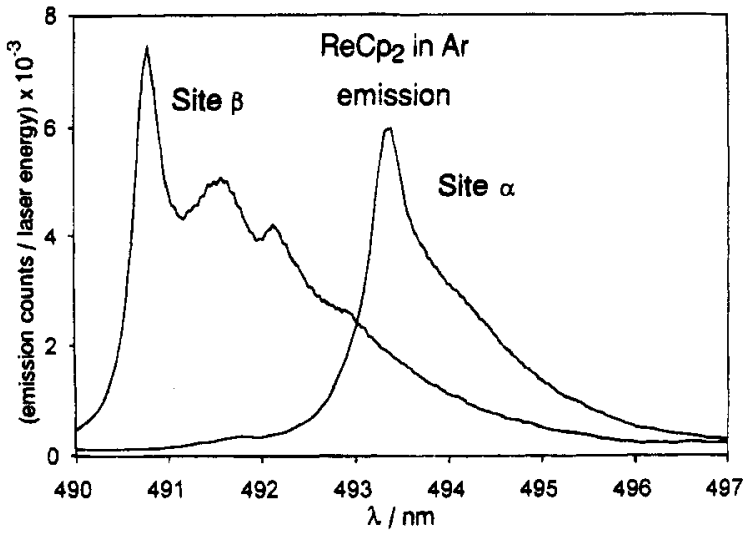

Figure 7. Expanded emission spectra of $\mathrm{ReCp}_{2}$ in an argon matrix showing just the $(0,1)$ transition. The two principal sites may be excited selectively: site $\alpha, \lambda_{\mathrm{ex}}=477.6 \mathrm{~nm}$, maximum $\lambda_{\mathrm{em}}=493.4 \mathrm{~nm}$; site $\beta$, $\lambda_{\mathrm{ex}}=475.3 \mathrm{~nm}$, maximum $\lambda_{\mathrm{em}}=490.8 \mathrm{~nm}$.

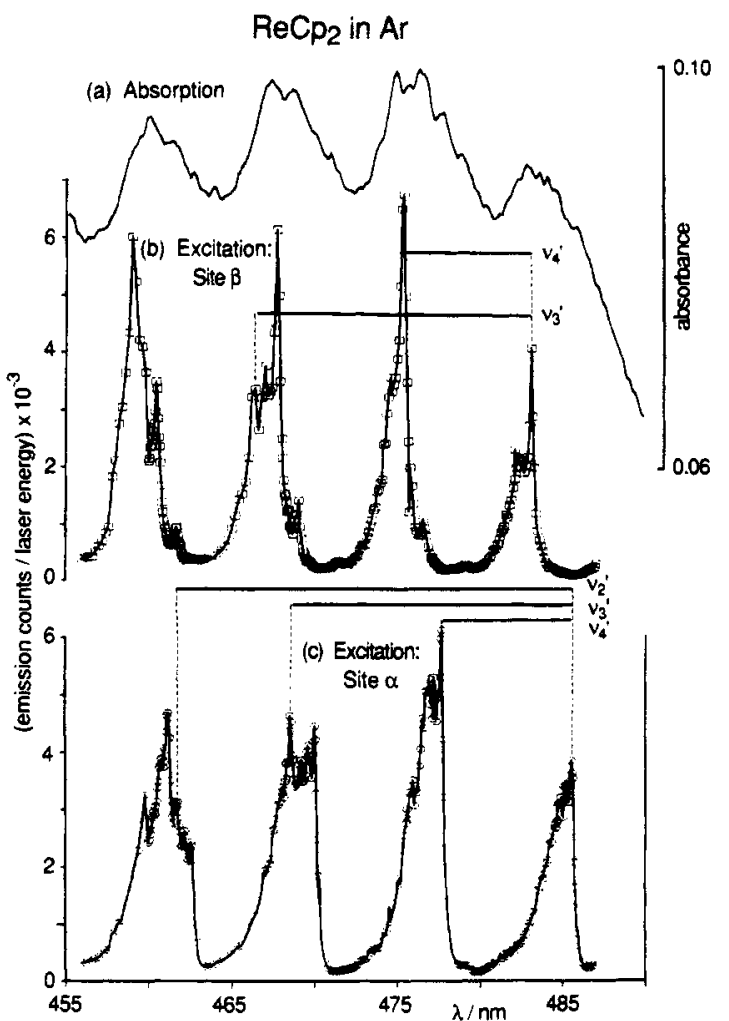

Figure 8. (a) Absorption spectrum of $\mathrm{ReCp}_{2}$ in an argon matrix. (b) Excitation spectrum of site $\beta, \lambda_{\mathrm{em}}=490.8 \mathrm{~nm}$. (c) Excitation spectrum of site $\alpha, \lambda_{\mathrm{em}}=493.4 \mathrm{~nm}$.

in the LIF in nitrogen matrices. The lack of complete selectivity in exciting the emission is apparent from Figure $1 \mathrm{~b}^{21}$

The intricate site structure is confirmed by variation of the excitation wavelength. In place of one major emission site as is seen in nitrogen, there are several in argon. Each site is probed to a different extent but not exclusively by the laser. Figure 7 shows the emission maxima chosen to construct excitation profiles for two sites, $\alpha$ and $\beta$. Each peak of Figure 7 corresponds to the $(0,1)$ emission and is chosen as it is well defined and significantly separated. Figure 8 shows the excitation profiles for sites $\alpha$ and $\beta$ with the absorption spectrum for comparison. Both excitation profiles show significantly better resolution than the absorption spectrum (Table VI, supplementary material). Three vibrational modes have been assigned for site $\alpha$ excitation. Only $\nu_{4}^{\prime}$ and $\nu_{3}^{\prime}$ can be located for site $\beta$. 
TABLE 3: Progression Frequencies $\left(\bar{v}, \mathrm{~cm}^{-1}\right)$ of $\mathrm{ReCl}_{2}$ in the Ground and Excited States with Standard Error $(95 \%$ Probability) in Parentheses, Where Appropriate

\begin{tabular}{lcccccc}
\hline matrix & $\bar{v}_{4}{ }^{\prime \prime}$ & $\bar{v}_{4}{ }^{\prime}$ & $\bar{v}_{3}{ }^{\prime \prime}$ & $\bar{v}_{3}{ }^{\prime}$ & $\bar{v}_{2}{ }^{\prime \prime}$ & $\bar{v}_{2}{ }^{\prime}$ \\
\hline Ar & $323(7)$ & $340(4)$ & 818 & 745 & 1097 & 1060 \\
$\mathrm{~N}_{2}$ sharp & $325(5)$ & $342(3)$ & 826 & 754 & 1100 & 1063 \\
$\mathrm{~N}_{2}$ broad & $330(8)$ & 341 & 828 & & &
\end{tabular}

The excited-state lifetimes of both site $\alpha$ and site $\beta$ have been examined. The lifetimes of site $\alpha$ are $69.3 \pm 1.4$ and $64 \pm 8$ ns, respectively, strikingly similar to the value determined in nitrogen matrices.

\section{Discussion}

1. Fluorescence of Rhenocene. The use of a pulsed tunable laser has allowed us to record sharp, well-resolved emission spectra for rhenocene. By exploiting the selectivity of the laser, well-defined excitation spectra have been recorded. The excitation spectra can be considered as experimental deconvolutions of the inhomogeneously broadened absorption spectra. The criteria for distinguishing resonance fluorescence and resonance Raman spectra in the gas phase have been defined very clearly by Ziegler. ${ }^{2} \mathrm{He}$ has also shown that relatively small changes in conditions can alter the balance between the two effects. Spectra of thenocene which are essentially identical in absolute frequency and intensity profile may be obtained at a variety of excitation wavelengths, if equivalent positions within the band envelope are probed. We have never observed any spectra which track with excitation frequency, the key criterion for resonance Raman spectroscopy. Moreover, the emission lifetime proves to be as long as $70 \mathrm{~ns}$. Thus, we invariably observe fluorescence from rhenocene, not resonance Raman scattering. In contrast, the $\mathrm{d}^{5} \operatorname{bis}\left(\eta^{6}\right.$-arene $)$ complexes of vanadium show resonance Raman scattering ${ }^{22}$ and not fluorescence. Whether the difference arises from the change in electronic ground state is not known (the vanadium complexes have $\mathrm{a}^{2} \mathrm{~A}_{1}$ ground state.)

2. Vibrational Fine Structure. The spectra have yielded the frequencies of three totally symmetric fundamental vibrations in ground and excited states. Mean values are presented in Table 3. The values of $v_{4}{ }^{\prime}$ and $v_{4}{ }^{\prime \prime}$ are essentially independent of the site and show little dependence on matrix material.

The ring-metal-ring stretching frequency, $v_{4}$, of $\mathrm{ReCp}_{2}$ is larger in the excited state than it is in the ground state. There is a substantial decrease in the $\nu_{3}$ frequency in the excited state and a smaller decrease in $v_{2}$. These changes reflect changes in the overall bonding in the molecule. The LMCT transition probed in the spectra formally transfers an electron from an $\mathrm{e}_{1}{ }^{\prime}$ orbital ( $D_{5 h}$ symmetry), which is metal-ligand nonbonding but $\pi$-bonding within the ligand, ${ }^{3,47,23}$ In the upper state this electron occupies an $e_{2}^{\prime}$ metal d orbital, which has significant metal-ligand bonding character. Removal of an electron from the $e_{1}{ }^{\prime}$ orbital will cause a reduction in the intra-ring bonding, and hence the value of $v_{2}$, the ring breathing mode, would be expected to be lower in the excited state. Placing the electron in the bonding $\mathrm{e}_{2}^{\prime}$ orbital will enhance the metal-ring interaction in the excited state, so causing the observed shift to higher frequency in $v_{4}$. (For comparison, $v_{4}$ is substantially reduced in the ligand field excited state of $\mathrm{RuCp}_{2}$, since the electron is transferred to the metal-ligand antibonding $e_{1}{ }^{\prime \prime}$ orbital. $)^{15,16}$ Such an approach does not explain why there is such a large change in the $\mathrm{C}-\mathrm{H}$ deformation mode, $\nu_{3}$.

From vibrational studies of stable metallocenes, the $\mathrm{C}-\mathrm{H}$ bending modes are most sensitive with respect to the nature of the metal and the character of the bonding. ${ }^{10}$ Analysis of the normal coordinates of $\mathrm{FeCp}_{2}$ and other metallocenes has shown that the contribution of the metal-ligand coordinate in the
$\delta(\mathrm{C}-\mathrm{H})$ modes is significant. A change in coupling of $\nu$ (ring$\mathrm{M}$-ring) to $\delta_{\text {sym }}(\mathrm{C}-\mathrm{H})$ in the excited state of $\operatorname{ReCp}_{2}$ may account for the larger change in $\nu_{3}$ than expected.

3. Inhomogeneous Broadening and Matrix Effects. The experiments have shown that the absorption spectrum of rhenocene in nitrogen matrices contains at least two independent components, whereas the emission and excitation spectra are selective (see Figure 4). These observations are inconsistent with multiple electronic origins as an explanation of the splitting but are consistent with the presence of multiple conformers or trapping sites. Even though the selectivity is incomplete in argon matrices, the same argument applies. The observation that the lifetime of the excited state changes by $\leqslant 15 \%$ between sites/conformers indicates that any perturbation by the matrix is small. Until comparable spectra are available from other phases, it will not be possible to distinguish the effect of trapping sites from the effects of conformation.

When individual bands are examined in argon and nitrogen matrices, we find that the emission and excitation spectra of $\mathrm{ReCp}_{2}$ are essentially mirror images, but highly asymmetric. In nitrogen, the half-width on the steep side is ca. $5 \mathrm{~cm}^{-1}$, while on the broad side the half-width is ca. $14 \mathrm{~cm}^{-1}$ and the bands tail out as much as $100 \mathrm{~cm}^{-1}$ beyond the maximum. The difference between the origins in excitation and emission is negligible. We now consider contributions to the band shapes from several effects: (i) phonon sidebands; (ii) matrix trapping sites and metallocene conformers; (iii) other low-frequency vibrational modes.

The phonons of solid nitrogen occur between 40 and 80 $\mathrm{cm}^{-1} .{ }^{24}$ In the simplest scenario, a phonon mode progression is excited. However, if there are several phonon modes that can couple to the electronic transition, a single sharp zero phonon line (ZPL) may be observed with a continuous phonon sideband. ${ }^{25}$ This model can explain the asymmetry of the observed band shape. The similarity of excitation and emission line shapes suggests that there is no difference between the ground and excited states in phonon coupling. This observation also fits with the experimentally coincident positions of the $(0,0)$ bands in the emission and excitation spectra.

The effect of multiple sites/conformers can manifest itself in the band shape through nonresonant excitation. Here, we would be concerned with sites with absorptions within $20 \mathrm{~cm}^{-1}$ of one another. When one site is irradiated resonantly into its $\mathrm{ZPL}$, other sites will be excited in their phonon wing. The net effect will be to add further intensity to the observed phonon wing of the resonantly excited site and smear any discernible phonon structure. This proposal does not offer an alternative explanation of the asymmetry of the bands, but can account for the slight changes in emission frequency which occur with small changes in excitation wavelength, especially in argon matrices.

Finally, we consider contributions of low-frequency intramolecular vibrations to the band shape. The low-frequency bending mode, $v_{22}$, of metallocenes cannot contribute since it occurs in the range $160-180 \mathrm{~cm}^{-1}$ and is non-totally symmetric. ${ }^{8-10}$ The torsional mode, $v_{7}$, of ferrocene has been identified by far IR spectroscopy at $44 \mathrm{~cm}^{-1}$ (cf. $66 \mathrm{~cm}^{-1}$ for $\mathrm{Cr}\left(\eta^{6}-\mathrm{C}_{6} \mathrm{H}_{6}\right)_{2}$ by inelastic neutron scattering). ${ }^{26,27}$ Thus the first overtone of the torsional mode of rhenocene could contribute to the asymmetric band shape in a similar way to the phonon modes. The band shapes in the LIF spectra of $\mathrm{MoCp}_{2}$ and $\mathrm{WCp}_{2}$ are noticeably more symmetrical than those for $\mathrm{ReCp}_{2}$-the reasons for this difference remain uncertain. ${ }^{28}$

4. Excited-State Lifetime. Measurements of the excitedstate decay kinetics for rhenocene reveal single-exponential 
behavior. The lifetime is close to 70 ns regardless of matrix material, site in the matrix, or matrix dilution. The lifetime is consistent with a fully allowed transition.

\section{Conclusions}

The fluorescence spectra of rhenocene isolated in nitrogen and argon matrices have been excited with a pulsed tunable laser. The emission and excitation spectra obtained are selective for individual sites/conformers in the matrix enabling us (i) to make precise measurements of the changes in vibrational frequency of three totally symmetric fundamental vibrations in the excited state, (ii) to measure a Franck-Condon intensity profile for emission and excitation, and (iii) to measure the excited-state lifetime ( $72 \mathrm{~ns}$ in solid nitrogen). In the excited state, there is an increase in the ring-metal-ring stretching mode, $v_{4}$, and a decrease in the C-H deformation, $v_{3}$, and in the ring breathing mode, $\nu_{2}$. These changes are consistent with the changes in bonding expected in this LMCT transition. The measurements provide the data necessary to estimate the change in geometry in the excited state-this will be the subject of a later paper. The technique of LIF offers considerable promise for the determination of structural information on organometallic transients both in the matrix and in fluid phases. In the succeeding paper, we extend the observations to molybdenocene and tungstenocene. ${ }^{28}$

Acknowledgment. We are particularly grateful to Dr. A. W. Parker and S. Tavender of the Rutherford-Appleton Laboratory for their support. We also appreciate the help given by Dr. S. E. J. Bell, S. A. Brough, and Dr. A. McCamley. This work was supported by SERC, the Royal Society, British Gas and the European Commission.

Supplementary Material Available: Tables III-VI: Emission spectra of $\mathrm{ReCp}_{2}$ from broad site in nitrogen matrix, absorption, emission, and excitation spectra in argon matrices ( 5 pages). Ordering information is given on any current masthead page.

\section{References and Notes}

(1) Present address: Department of Chemistry, St. Patrick's College, Maynooth, Ireland.

(2) Bondybey, V. E. In Chemistry and Physics of Matrix-Isolated Species; Andrews, L., Moskovits, M., Eds.; North-Holland: Amsterdam, 1989. Perutz, R. N. Chem. Rev. 1985, 85, 97. Ziegler, L. D. Acc. Chem. Res. 1994, 27, 1.
(3) Cox, P. A.; Grebenik, P.; Perutz, R. N.; Graham, R. G.; Grinter, R. Chem. Phys. Lett. 1984, 108, 415. Chetwynd-Talbot, J.; Grebenik, P.; Perutz, R. N.; Powell, M. H. A. Inorg. Chem. 1983, 22, 1675.

(4) Bandy, J. A.; Cloke, F. G. N.; Cooper, G.; Day, J. P.; Girling, R. B.; Graham, R. G.; Green, J. C.; Grinter, R.; Perutz, R. N. J. Am. Chem. Soc. 1988, 110, 5039. 453.

(5) Grebenik, P.; Grinter, R.; Perutz, R. N. Chem. Soc. Rev. 1988, 17,

(6) Bell, S. E. J.; Hill, J. N.; McCamley, A.; Perutz, R. N. J. Phys. Chem. 1990, 94, 3876. Perutz, R. N.; Hill, J. N.; McCamley, A. Coord Chem. Rev. 1991, 111, 111.

(7) Graham, R. G.; Grinter, R.; Perutz, R. N. J. Am. Chem. Soc. 1988 $110,7036$.

(8) Lippincott, E. R.; Nelson, R. D. Spectrochim. Acta 1958, $10,307$.

(9) Bodenheimer, J. S.; Low, W. Spectrochim. Acta 1973, 29A, 1733.

(10) Aleksanyan, V. T. In Vibrational Spectra and Structure; Durig, J. R., Ed.; Elsevier: Amsterdam, 1982; Vol. 11.

(11) The IR frequencies of $\mathrm{ReCp}_{2}$ in $\mathrm{N}_{2}$ matrices are $1102\left(\mathrm{a}_{2 \mathrm{u}}\right), 820$ $\left(\mathrm{a}_{2 \mathrm{u}}\right), 316\left(\mathrm{a}_{2 \mathrm{u}}\right), 977\left(\mathrm{e}_{\mathrm{tu}}\right)$, and $298 \mathrm{~cm}^{-1}\left(\mathrm{e}_{1 \mathrm{u}}\right)^{2}$

(12) Crosby, G. A.; Hager, G. D.; Hipps, K. W.; Stone, M. L. Chem. Phys. Lett. 1974, 28, 497.

(13) Wrighton, M. S.; Pdungsap, L.; Morse, D. L. J. Phys. Chem. 1975, 79,66 .

(14) Hipps, K. W.; Francis, A. H. J. Phys. Chem. 1979, 83, 1879.

(15) Hollingsworth, G. J.; Kim Shin, K.-S.; Zink, J. I. Inorg. Chem. $1990,29,2501$.

(16) Riesen, H.; Krausz, E.; Luginbühl, W.; Biner, M.; Güdel, H. U.; Ludi, A. J. Chem. Phys. 1992, 96, 4131.

(17) Robles, E. S. J.; Ellis, A. M.; Miller, T. A. J. Phys. Chem. 1992, $96,3247$.

(18) Robles, E. S. J.; Ellis, A. M.; Miller, T. A. J. Phys. Chem. 1992, 96, 8791; J. Am. Chem. Soc. 1992, 114, 7171.

(19) (a) Lees, A. J. Chem. Rev. 1987, 87, 711. (b) Paulson, S.; Sullivan, B.: Caspar, J. V. J. Am. Chem. Soc. 1992, 114, 6905. Kenney, J. W., Boone, D. R.; Striplin, D. R.; Yong, H.; Hamar, K. B. Organometallics 1993, 12, 3671 .

(20) Green, M. L. H.; Pratt, L.; Wilkinson, G. J. Chem. Soc. 1958, 3916.

(21) While the A progression dominates the spectrum of $\mathrm{ReCp}_{2}$ in argon, there are some extra very weak features not associated with the $A$ series. There is a small feature located at $20775 \mathrm{~cm}^{-1}$, ca. $70 \mathrm{~cm}^{-1}$ to higher frequency than the $(0,0)$ band observed in the absorption spectrum and 180 $\mathrm{cm}^{-1}$ from $\mathrm{A} 0$. There is also a minor progression with origin $114 \mathrm{~cm}^{-1}$ to high frequency of the A series.

(22) McCamley, A.; Perutz, R. N. J. Phys. Chem. 1991, 95, 2739

(23) Warren, K. D. Structure Bonding (Berlin) 1976, 27, 45. Green, J. C. Ibid. 1981, 43, 37 .

(24) Knözinger, E.; Wittenbeck, R. J. Am. Chem. Soc. 1983, 105, 2154.

(25) Rebane, K. Impurity Spectra of Solids; Plenum: New York, 1970; J. Am. Chem. Soc. 1983, 105, 2154.

(26) Rocquet, F.; Berreby, L.; Marsault, J. P. Spectrochim. Acta 1973, $29 A, 1101$.

(27) Howard, J., Robson, K., Waddington, T. C. J. Chem. Sac., Dalton Trans. 1982, 977 .

(28) Hill, J. N.; Perutz, R. N.; Rooney, A. D. Following paper in this issue. 